Meta

Journal des traducteurs

Translators' Journal

\title{
Projet de dictionnaire interactif multilingue de termes médicaux
}

\section{Maria de Lurdes Abrantes Garcia}

Volume 42, numéro 1, mars 1997

Lexicologie et terminologie

URI : https://id.erudit.org/iderudit/003524ar

DOI : https://doi.org/10.7202/003524ar

Aller au sommaire du numéro

Éditeur(s)

Les Presses de l'Université de Montréal

\section{ISSN}

0026-0452 (imprimé)

1492-1421 (numérique)

Découvrir la revue

Citer cet article

de Lurdes Abrantes Garcia, M. (1997). Projet de dictionnaire interactif multilingue de termes médicaux. Meta, 42(1), 110-113.

https://doi.org/10.7202/003524ar

\section{Résumé de l'article}

L'eclosión des nouvelles technologies a été un événement décisif pour le développement du génie linguistique, spécialement pour la fabrication des outils didactiques d'apprentissage des langues étrangères. L'existence d'un dictionnaire spécialisé en langues scientifique ou technique peut contribuer à un apprentissage des aspects lexicologiques et terminologiques et assurer une acquisition plus efficace d'une langue étrangère. C'est l'objectif prioritaire de notre recherche. Nous présentons notre projet de fabrication d'un dictionnaire interactif multilingue (portugais, français et anglais) de termes médicaux dans le domaine de la sénologie (maladies du sein). Nous décrivons quelques aspects de la méthodologie utilisée, assistée par des outils informatisés, tels que les bases de données textuelles et terminologiques. 


\title{
PROJET DE DICTIONNAIRE INTERACTIF MULTILINGUE DE TERMES MÉDICAUX*
}

\author{
MARIA DE LURDES ABRANTES GARCIA 1
}

Universidade Nova de Lisbou, Lisbonne, Portugal

\begin{abstract}
Résumé
L'éclosion des nouvelles technologies a été un événement décisif pour le développement du génie linguistique, spécialement pour la fabrication des outils didactiques d'apprentissage des langues étrangères. L'existence d'un dictionnaire spécialisé en langues scientifique ou technique peut contribuer à un apprentissage des aspects lexicologiques et terminologiques et assurer une acquisition plus efficace d'une langue étrangère. C'est l'objectif prioritaire de notre recherche.

Nous présentons notre projet de fabrication d'un dictionnaire interactif multilingue (portugais, français et anglais) de termes médicaux dans le domaine de la sénologie (maladies du sein). Nous décrivons quelques aspects de la méthodologie utilisée, assistée par des outils informatisés, tels que les bases de données textuelles et terminologiques.
\end{abstract}

\begin{abstract}
The advent of new technologies has been a pivotal factor in the developmerit of language engineering especially with regard to producing tools for foreign language acquisition. Specialized technical dictionaris can foster more effective learning of lexicon and terminology and enhance foreign language acquisition. This article presents our multilingual (Portuguese, French and English) interactive medical dictionary in the area of senology, and describes a computer-assisted approach using text and terminology databases.
\end{abstract}

\section{Resumo}

O desenvolvimento das novas tecnologias tornou-se um acontecimento decisivo para o desenvolvimento da Engenharia Linguistica, nomeadamente para a produção de instrumentos de importância vital para a aprendizagem de linguas. A existência de um dicionário de uma língua técnica pode promover uma aprendizagem mais eficaz de conteudos lexicais e terminológicos, contribuindo para uma melhor comunicaşão e aprendizagem de línguas.

Pretendemos assim apresentar o nosso projecto relativo à elaboração de um dicionário interactivo Multilingue (Português, Francês e Inglês) de termos Médicos no domínio da Senologia, referindo a sua metodologia baseada em instrumentos informatizados - bases de dados textuais e terminológicos.

La variété et la richesse des langues des États membres de l'Union européenne constituent une motivation pour une collaboration plus étroite entre terminologues, lexicographes, traducteurs et groupes de scientifiques, en particulier les médecins.

Notre recherche a commc objectif l'élaboration d'un dictionnaire interactif multilingue (portugais, français et anglais) de termes médicaux dans le domaine de la sénologie. Nous travaillons en collaboration avec plusieurs professeurs de médecine des universités de Lisbonne, de Coimbra et de Porto.

Les membres de l'équipe se préoccupent particulièrement de l'établissement d'une néologie raisonnée (Quemada 1971), problématique toujours présente pour l'harmonisation terminologique (termes et définitions) par consensus entre spécialistes.

La sénologie est une branche et une spécialité des sciences médicales qui propose une approche multidisciplinaire du traitement de la glande mammaire et de sa pathologie, 
en des domaines aussi diversifiés que la recherche de base et la recherche clinique, la prévention, le dépistage, le diagnostic, le traitement, la réhabilitation et l'assistance aux patientes en phase terminale.

Le cancer du sein est une pathologie d'une grande importance, il a une grande incidence chez la femme dans la plupart des pays occidentaux, et est responsable d'un grand pourcentage de décès (il représente $26 \%$ du total des cancers féminins).

Les terminologues peuvent aider les sénologues dans leur travail en contribuant à l'amélioration de leur compétence communicative, dont la précision terminologique est partie intégrante.

Les questions terminologiques, indissociables de toutes les problématiques sénologiques, se présentent souvent comme une des préoccupations des auteurs d'ouvrages et d'études scientifiques publiés dans ce domaine.

Bref, l'objectif de notre recherche, dans un premier temps, est l'étude de la «sénoterminologie» (terminologie de la sénologie) pour le projet de lexicographie informatisée de spécialité. Ce travail comprend une composante terminodidactique, c'est-à-dire qu'il doit contribuer à l'acquisition/apprentissage des étudiants de médecine portugais ou étrangers de la terminologie de cette langue de spécialité; il doit spécifier des aspects particuliers de caractère lexicologique, terminologique, phraséologique pour les traducteurs. Cette approche terminodidactique permet d'offrir à l'utilisateur des outils d'aide à la compréhension et à la production d'énoncés corrects et cohérents dans ce domaine de connaissance.

Cette étude terminologique nous permet d'analyser le grand nombre de néonymes du domaine (néologismes scientifiques), l'ensemble des termes qui vieillissent et les définitions qui présentent des phénomènes de changement. Cela est dû aux progrès scientifiques et au statut interdisciplinaire de la sénologie.

Cependant, la productivité lexicale et/ou terminologique n'est pas toujours synonyme de précision et de communication efficace; il est impératif d'uniformiser, de supprimer les polysémies et de désambiguïser cette terminologie ; mais cette action normalisatrice doit se faire dans une perspective de «néologie raisonnée».

B. Quemada (1971: 137-150), considérant la valeur de la formation et de l'information lexicologique/terminologique pour les créateurs de terminologie, met en évidence l'importance de la «néologie raisonnée». Il considère que dans une langue de culture moderne, nécessairement scientifique et technique, la néologie lexicale est la condition pour que cette langue puisse continuer à être un outil de communication national et conserver le statut de langue vivante. Donc, la néologie est nécessaire à la vitalité d'une langue.

Dans les vocabulaires techniques-scientifiques, l'usage est «le juge» et «l'arbitre suprême», parce qu'il est «une synthèse de forces très diversifiées issues d'impulsions psychologiques, économiques, culturelles, des règles d'économie fonctionnelle de la communication, etc., autant de facteurs qui conditionnent l'apparition, la diffusion et les chances de survie d'un néologisme» (Quemada 1971: 141).

Un ensemble de facteurs et de circonstances favorables détermine l'implantation d'un terme donné, dans la plupart des usages.

Le «dirigisme linguistique» est inacceptable dans la réalisation d'un projet d'uniformisation terminologique; nous préférons la perspective de $\mathrm{B}$. Quemada qui privilégie un programme d'«assistance néologique»; cela consiste dans l'accès aux termes par tous les moyens possibles, au public qui a besoin d'une assistance terminologique, de façon à concrétiser les exigences de précision du discours scientifique ou de spécialité. Notre projet privilégie la formation et un accès rapide à l'information qui doit contribuer à la régularisation et à l'harmonisation terminologiques établies par des consensus entre des 
sénologues et d'autres médecins spécialistes, avec l'aide du terminologue, c'est-à-dire de nous-même; nous comptons sur l'appui de l'Unité de Recherche de «Lexicologie, Lexicographie et Terminologie» de l'Université Nouvelle de Lisbonne.

Nous croyons, donc, que l'informatique, le génie linguistique, les nouvelles technologies de l'information et les réseaux télématiques sont indispensables à la concrétisation d'un programme d'«assistance néologique» associée à un projet de dictionnairique de spécialité.

L'organisation d'une base textuelle et d'une base de données terminographiques, deux bases complémentaires, permet l'enregistrement et l'observation de phénomènes néonymiques et terminologiques.

La base textuelle, gérée par l'hypertexte statistique Hyperbase (logiciel de E. Brunet), est constituée d'un corpus trilingue, formé de textes scientifiques de sénologie, thématiquement équivalents en portugais, en français et en anglais.

Le logiciel Hyperbase a permis plusieurs types d'analyses (cf. Garcia 1993: 4-5):

- l'extraction des unités terminologiques presque transparentes entre les trois langues, c'est-à-dire, des «internationalismes» terminologiques;

- la recherche de formants pour vérifier nos observations empiriques sur la fréquence des internationalismes au niveau morphologique, ainsi que leur productivité ;

- le listage de tous les formants qui entrent dans la constitution de cette terminologie et qui la caractérisent;

- le repérage de contextes de termes et de formants; donc des contextes de substance en ce qui concerne les termes; des contextes plutôt formels en ce qui concerne les particularités morphosémantiques des formants;

- la recherche de définitions dans les trois langues. Éventuellement, nous pouvons vérifier soit la coïncidence des définitions, soit quelques particularités relatives aux différentes conceptualisations existantes dans chacune des langues;

- l'étude d'équivalents en français et en anglais;

- l'extraction de concordances qui permettent l'étude des collocations de termes et des distributions des formants dans chacune des langues;

- l'étude du terme en discours: son fonctionnement syntaxique;

- l'étude de l'idiomaticité caractéristique de ce type de discours scientifique;

- la recherche en phraséologie contrastive de façon à décrire les particularités spécifiques à chacune des langues.

Une base de données terminologiques enregistre et systématise, en parallèle, les particularités terminologiques.

Le dictionnaire multilingue interactif de la sénologie doit être utile à plusieurs types de publics. D'abord aux sénologues, spécialistes du domaine qui ont souvent besoin de préciser le champ sémantique d'un terme employé dans plusieurs domaines d'applications. Par ailleurs, le spécialiste a besoin d'être au courant des néonymes, des changements de nomenclatures et de définitions dus à l'évolution constante de la science et de la technologie ; il doit connaître les équivalents; il a besoin de se familiariser avec les synonymes (de façon à éviter les faux synonymes), les caractéristiques discursives et communicatives des termes, les phraséologismes et l'orthographe correcte, chaque fois qu'il a besoin de rédiger un article soit dans sa langue maternelle, soit dans une langue étrangère.

Les médecins généralistes ou d'autres spécialités auront aussi à leur disposition des éléments utiles à 1 'élaboration de rapports précis sur les patients avec des symptômes de maladies sénologiques.

Les étudiants de médecine trouveront réponse à leurs doutes terminologiques et pourront consolider l'apprentissage de ce discours scientifique particulier. 
En conclusion, nous espérons que ce dictionnaire offrira des éléments utiles aux traducteurs et aux rédacteurs spécialisés.

\section{Notes}

* Este trabalho foi apoiado pelo Instituto Camões e pela JNICT através do Programa Lusitânia.

1. Cet article est issu d'une communication présentée par l'auteur aux IV Journées scientifiques du réseau «Lexicologie, terminologie, traduction» de l'AUPELF-UREF (Lyon, France, 28, 29, 30 septembre 1995).

\section{RÉFÉRENCES}

CONTENTE, M. et J. MAGALHÃES (1994) : «A Terminologia da Medicina - A Problemática dos Equivalentes», V Congresso Luso-Hispano de Lenguas Aplicadas a las Ciencias, Universidade de Valência, 29, 30 Set. 1 Oct., Actes à paraître.

GALISSON, R. (1987): «De la lexicographie de dépannage à la lexicographie d'apprentissage», Cahiers de lexicologie, 51 (2), Paris, Didier.

GALISSON, R. (1989): «Enseignement et apprentissage des langues et des cultures : 'évolution' ou 'révolution' pour demain?», A.E.G.L. Bilans et perspectives, vol. 1, Paris.

GARCIA, M. L. (1993): «Base de données textuelles - Corpora trilingue et études des internationalismes terminologiques de la sénologie», Revue Cumfid, Nice, U.F.R. Lettres, Arts et Sciences Humaines, Université de Nice.

GARCIA, M. L. (1994a) : «A Internacionalização da linguagem médica numa perspectiva de ensino de Lsp», $V$ Congresso Luso-Hispano de Lenguas Aplicadas a las Ciencias, Universidade de Valência, 29, 30 Set. 1 Oct., Actes à paraître.

GARCIA, M. L. (1994b): «A Terminologia Médica», IVo Simpósio Ibero Americano de Terminologia RITerm - Terminología e Desarrollo -, Buenos Aires, 17-21 Out. 1994, Actes à paraître.

GARCIA, M. L. (1994c) : Terminologia da Senologia - Projecto de um dicionário interactivo multilingue para uma aprendizagem da Lsp, Dissertação de Mestrado, Lisboa, F.C.S.H., Universidade Nova de Lisboa.

GARCIA, M. L. (1994d) : «Dicionário interactivo multilingue de Senologia - um contributo para aprendizagem de Terminologia Médica», Terminologias, 9, Lisboa, Termip, Associação de Terminologia Portuguesa.

GAUDIN, F. (1991): «Terminologie et transfert de connaissances : à propos de dictionnaires scientifiques», 3rd Infoterm Symporium, Infoterm.

LINO, M. T. (1992) : «Base de Dados Textuais - Portext. Lexicologia, Lexicografia e Terminologia», Terminologias, 5-6, Lisboa, Termip.

LINO, M. T. (1994a): «Base de données textuelles et terminographie», Mera, Hommage à Bernard Quemada, Montréal (Canada).

LINO, M. T. (1994b) : «Corpora informatisés et travail en terminographie: expérience portugaise», Alfa, $7 / 8$, Canáda, Université Dalhousie.

PORQUIER, R. et al. (1993): «Le statut des outils métalinguistiques dans l'apprentissage et l'enseignement au niveau avancé», Études de linguistique appliquée, no 92, Paris, Didier.

QUEMADA, B. (1971) : «À propos de la néologie», La Banque des Mots, 2, Paris, Cilf, PUF.

QUEMADA, B. (1987): «Notes sur lexicographie et dictionnairique», Cahiers de lexicologie, $\mathrm{n}^{\mathrm{a}}$ 51-2, Paris, Didier.

SCHMITT, C. (1991): «L'Europe et l'évolution des langues de specialité», Terminologie et traduction, $\mathrm{n}^{\circ} 2$, Commission des Communautés européennes. 